Electronic supplementary information for the paper

\title{
Predicting adsorption on metals: Simple yet effective descriptors for surface catalysis
}

Erik-Jan Ras, ${ }^{* a, b}$ Manuel J. Louwerse, ${ }^{a}$ Marjo C. Mittelmeijer-Hazeleger ${ }^{a}$ and Gadi Rothenberg ${ }^{a, *}$

${ }^{a}$ Avantium Technologies B.V. Zekeringstraat 29, 1014BV Amsterdam, The Netherlands. Tel. $+31(0) 20$ 586 8080, Fax. +31 (0)20 586 8085, email: erikjan.ras@avantium.com

${ }^{b}$ Van 't Hoff Institute for Molecular Sciences, University of Amsterdam, Science Park 904, 1098XH Amsterdam, The Netherlands. Tel. +31 (0)20 525 6963, Fax.+31 (0)20 525 5604, e-mail:

g.rothenberg@uva.nl http://www.science.uva.nl/hims/hcsc

This document contains the detailed experimental results, additional information on the models reported in the main text and information required to reproduce the reported models. Specifically, all measured adsorption terms are given, the detailed equations of the obtained models, a separate set of models for hydrogen and hydroxyl radical and details on the specific variables and applications included in each model are provided. The additional information provided for the modeling results are sufficient to provide the reader with the means to reproduce any model reported in the main text.

\section{Measured adsorption isotherms}

This section contains all measured adsorption isotherms for the gases $\left(\mathrm{CH}_{4}, \mathrm{CO}, \mathrm{CO}_{2}, \mathrm{H}_{2}, \mathrm{~N}_{2}\right.$, and $\left.\mathrm{O}_{2}\right)$ and solid materials (bare $\mathrm{TiO}_{2}$ and $1 \mathrm{wt} \% \mathrm{Ni}, \mathrm{Pt}$ and $\mathrm{Rh}$ on the same $\mathrm{TiO}_{2}$ ) investigated. The data is grouped per adsorptive gas $\left(\mathrm{CH}_{4}\right.$ in Figure $\mathrm{S} 1, \mathrm{CO}$ in Figure $\mathrm{S} 2, \mathrm{CO}_{2}$ in Figure $\mathrm{S} 3, \mathrm{H}_{2}$ in Figure $\mathrm{S} 4, \mathrm{~N}_{2}$ in Figure $\mathrm{S} 5$ and $\mathrm{O}_{2}$ in Figure S6). The separate plots allow for the widely different scales at which the adsorption events take place. 

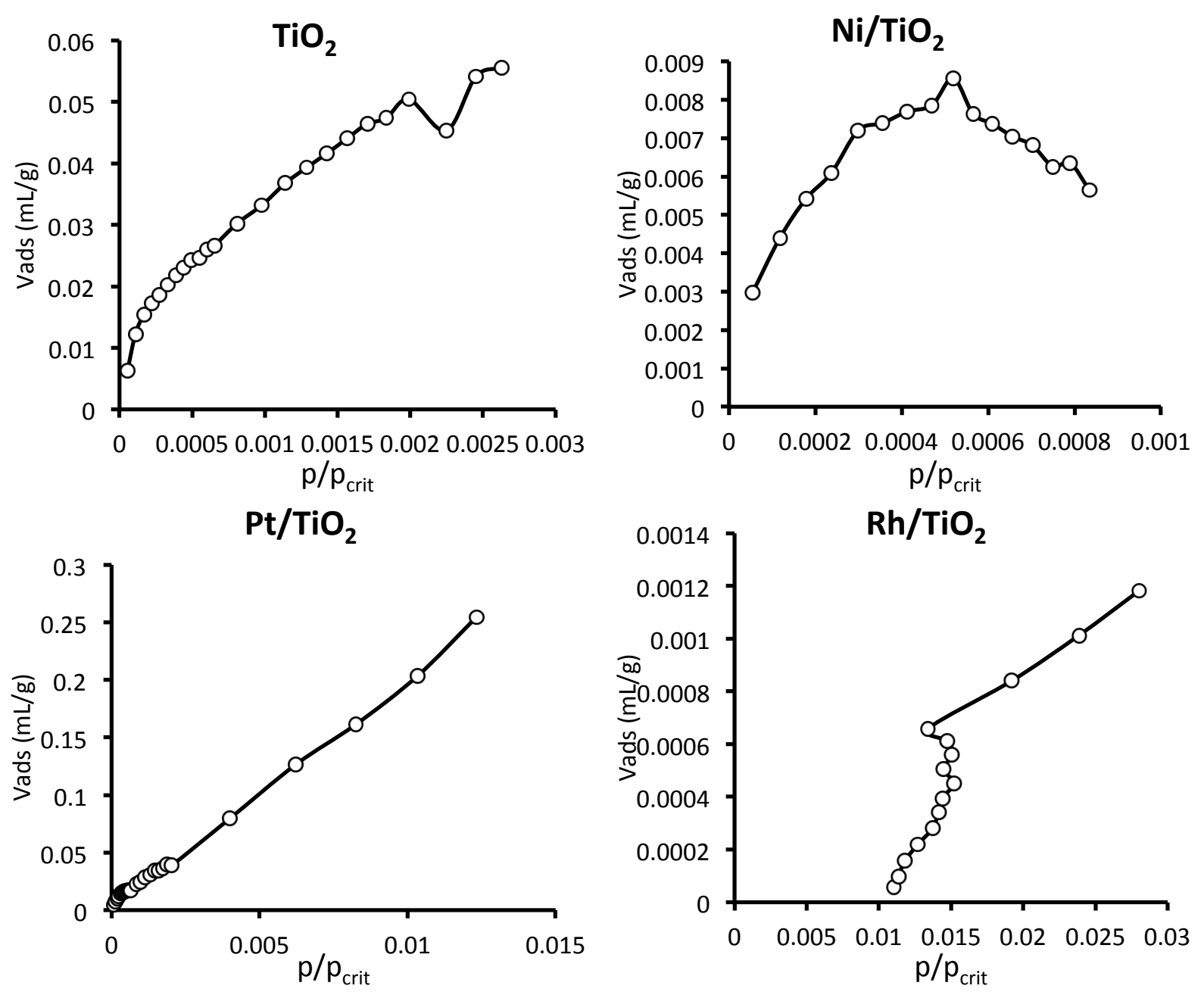

Figure $\mathrm{S} 1$ Measured adsorption isotherms for $\mathrm{CH}_{4}$, as adsorbed volume per unit mass of solid versus $\mathrm{p} / \mathrm{p}_{\text {crit, }}$ for all 4 solid materials (bare $\mathrm{TiO}_{2}$ and $1 \mathrm{wt} \% \mathrm{Ni}$, $\mathrm{Pt}$ and $\mathrm{Rh}$ on the same $\mathrm{TiO}_{2}$ ). 

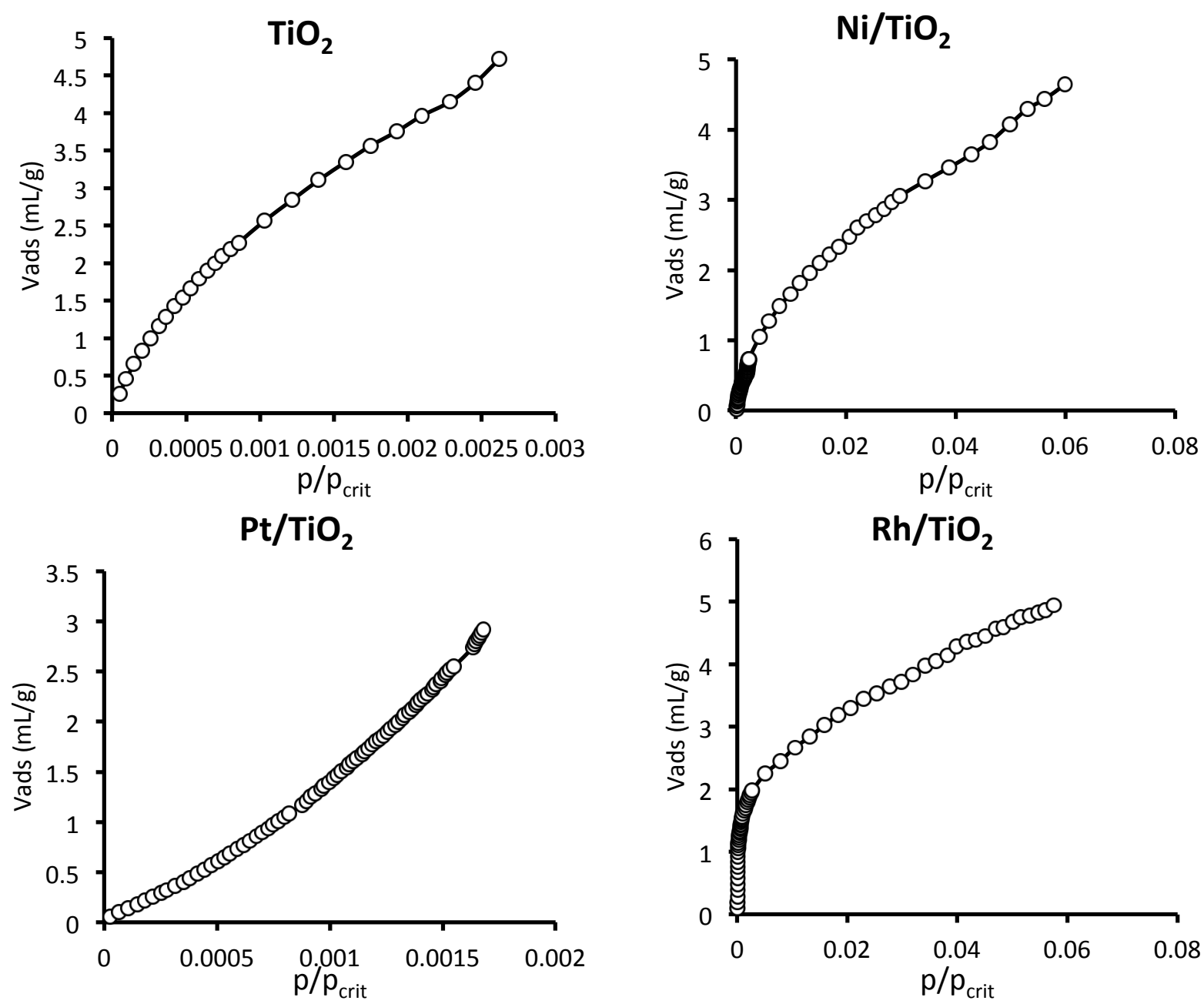

Figure S2 Measured adsorption isotherms for $\mathrm{CO}$, as adsorbed volume per unit mass of solid versus $p / p_{\text {crit, }}$, for all 4 solid materials (bare $\mathrm{TiO}_{2}$ and $1 \mathrm{wt} \% \mathrm{Ni}, \mathrm{Pt}$ and $\mathrm{Rh}$ on the same $\mathrm{TiO}_{2}$ ). 

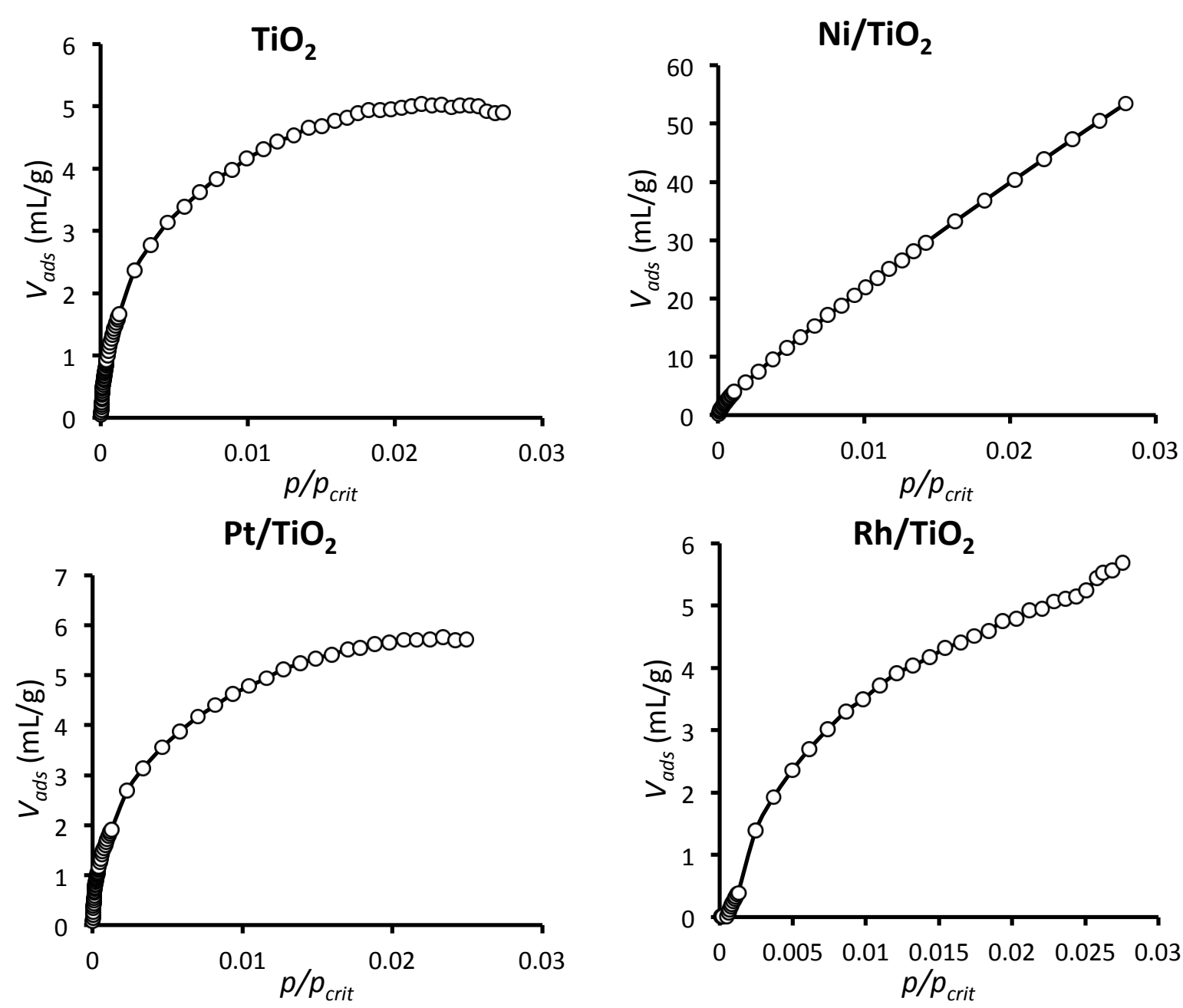

Figure S3 Measured adsorption isotherms for $\mathrm{CO}_{2}$, as adsorbed volume per unit mass of solid versus $p / p_{\text {crit, }}$, for all 4 solid materials (bare $\mathrm{TiO}_{2}$ and $1 \mathrm{wt} \% \mathrm{Ni}$, Pt and $\mathrm{Rh}$ on the same $\mathrm{TiO}_{2}$ ). 

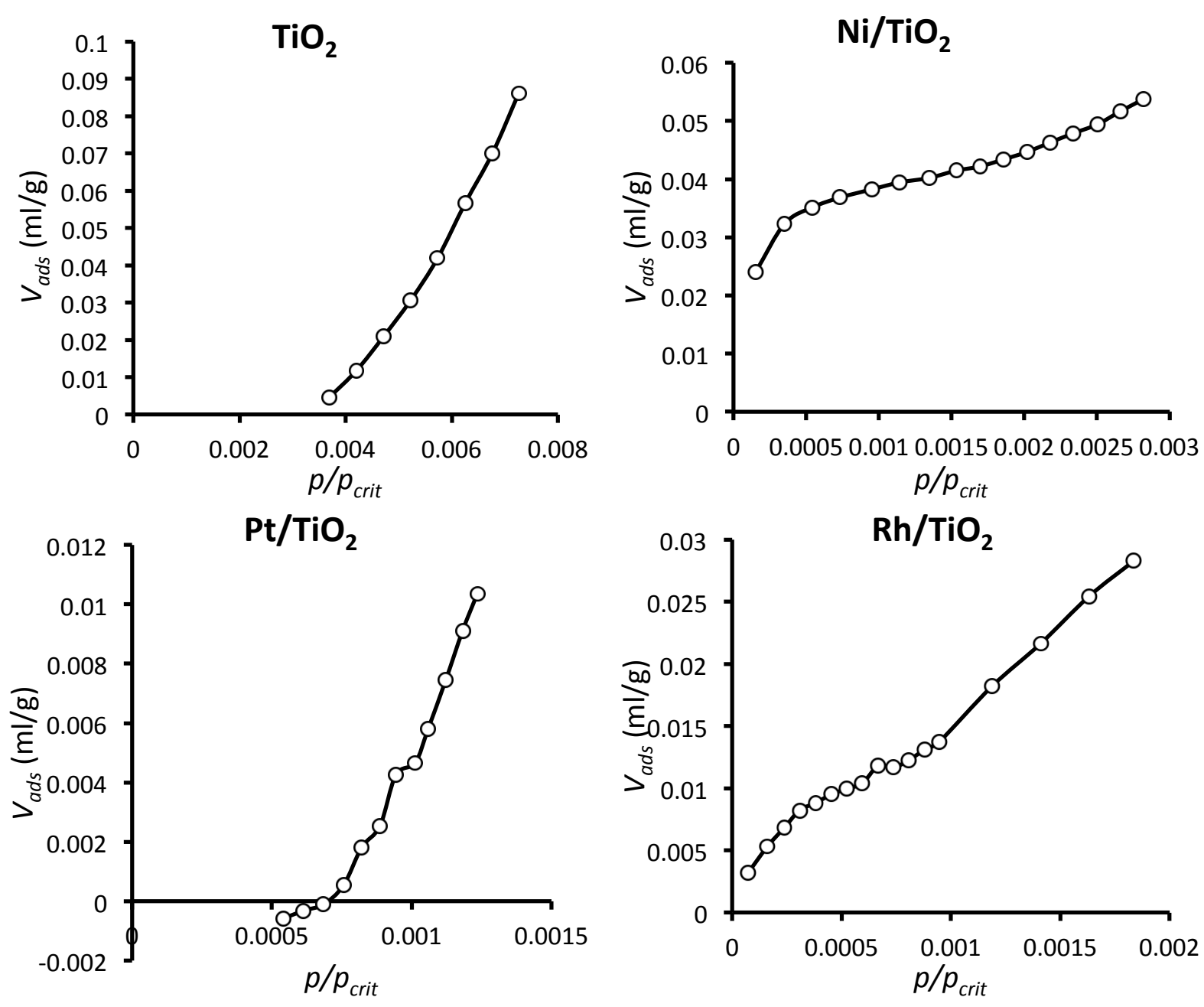

Figure S4 Measured adsorption isotherms for $\mathrm{H}_{2}$, as adsorbed volume per unit mass of solid $p / p_{\text {crit, }}$, for all 4 solid materials (bare $\mathrm{TiO}_{2}$ and $1 \mathrm{wt} \% \mathrm{Ni}$, Pt and $\mathrm{Rh}$ on the same $\mathrm{TiO}_{2}$ ). 

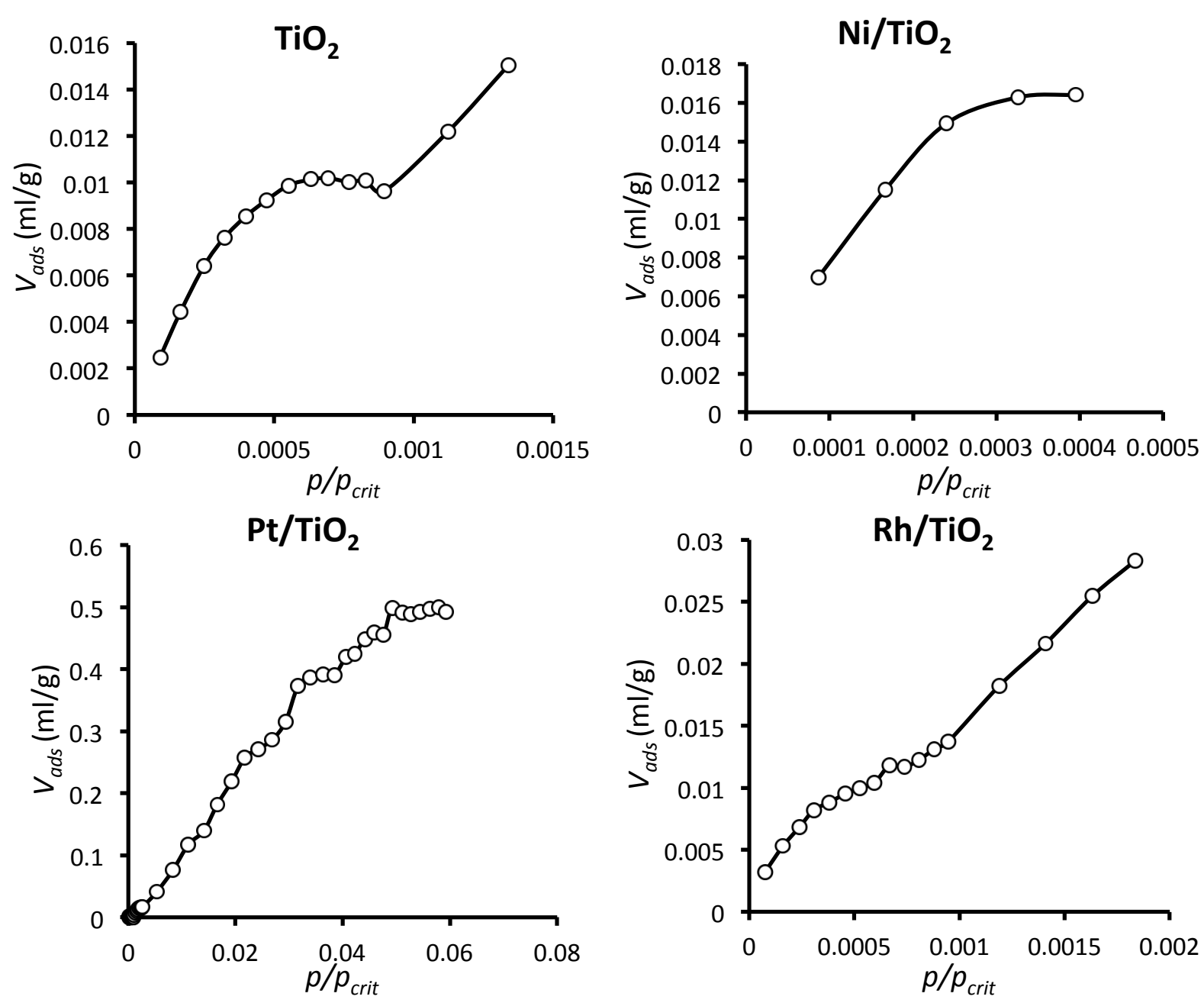

Figure S5 Measured adsorption isotherms for $\mathrm{N}_{2}$, as adsorbed volume per unit mass of solid versus $p / p_{\text {crit, }}$ for all 4 solid materials (bare $\mathrm{TiO}_{2}$ and $1 \mathrm{wt} \% \mathrm{Ni}, \mathrm{Pt}$ and $\mathrm{Rh}$ on the same $\mathrm{TiO}_{2}$ ). 

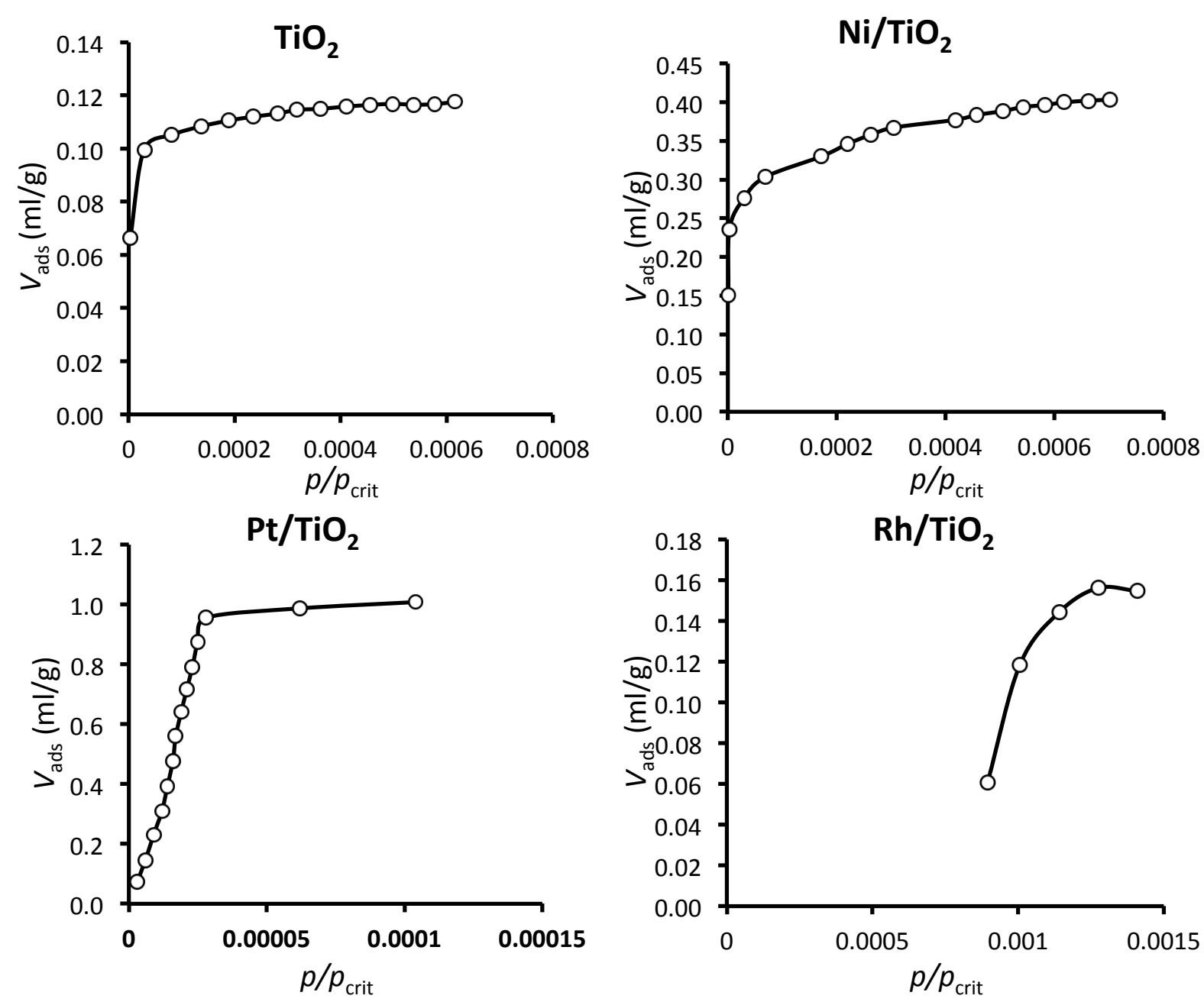

Figure S6 Measured adsorption isotherms for $\mathrm{O}_{2}$, as adsorbed volume per unit mass of solid versus $p / p_{\text {crit, }}$ for all 4 solid materials (bare $\mathrm{TiO}_{2}$ and 1 wt\% Ni, Pt and Rh on the same $\mathrm{TiO}_{2}$ ).

\section{Equations for best models}

Below the coefficients are given for the best models (out of the 27 possibilities, from A1B1C1 to A3B3C3) based on DFT data as indicated in Table 3 in the main text. Each table gives the variable identifier, the scaled model coefficients for comparing the importance of each parameter within a model and the real model coefficients to apply the model to calculate heat of adsorption values. As an example, the full equation for model A1B2C1:

$$
\begin{gathered}
\Delta H_{\text {ads }}=-7.3122-2.4519 \cdot x_{4}-0.0860 \cdot x_{5}+0.0296 \cdot x_{7}+0.0427 \cdot x_{1} \cdot x_{2}+0.0115 \cdot x_{1} \cdot x_{9}-0.0150 \cdot x_{2} \\
\cdot x_{7}-0.0311 \cdot x_{2} \cdot x_{9}+0.1591 \cdot x_{4} \cdot x_{8}+0.0012 \cdot x_{5} \cdot x_{6}+0.0018 \cdot x_{7} \cdot x_{8}
\end{gathered}
$$


Table S1 Scaled and real coefficients for the best model obtained for the full data set (A1B2C1).

\begin{tabular}{ccc}
\hline Parameter & $\begin{array}{c}\text { Scaled } \\
\text { coefficient }\end{array}$ & Real coefficient \\
\hline Intercept & -0.0918243 & -7.31229 \\
x4 & -0.0709649 & -2.45185 \\
x5 & -0.0595861 & -0.0859984 \\
x7 & 0.256484 & 0.0296089 \\
x1*x2 & 0.0463655 & 0.0427118 \\
x1*x9 & 0.458749 & 0.0115422 \\
x2*x7 & -0.531661 & -0.0150488 \\
x2*x9 & -0.364033 & -0.0311441 \\
x4*x8 & 0.234732 & 0.159055 \\
x5*x6 & 0.244552 & 0.0012333 \\
x7*x8 & 0.390236 & 0.0018444 \\
\hline
\end{tabular}

Table S2 Scaled and real coefficients for the best model obtained for the data set excluding $\mathrm{H}_{2}(\mathrm{~A} 2 \mathrm{~B} 2 \mathrm{C} 1)$.

\begin{tabular}{ccc}
\hline Parameter & $\begin{array}{c}\text { Scaled } \\
\text { coefficient }\end{array}$ & Real coefficient \\
\hline intercept & -0.0263352 & -9.89117 \\
x7 & 0.0486255 & 0.0091438 \\
$x 9$ & -0.0319799 & -0.0111913 \\
x1*x7 & 0.286961 & 0.0031395 \\
x1*x9 & 0.174436 & 0.0062212 \\
x2*x5 & -0.291259 & -0.122037 \\
x2*x9 & -0.231181 & -0.0255706 \\
x4*x7 & 0.0750419 & 0.0093469 \\
$x 4 * x 8$ & 0.140208 & 0.108952 \\
x4*x9 & -0.0232902 & -0.0058702 \\
x5*x7 & 0.0888695 & 0.0008441 \\
$x 5 * x 8$ & 0.13099 & 0.0082944 \\
$x 6 * x 7$ & 0.112894 & $5.19 \mathrm{E}-05$ \\
$x 8 * x 9$ & 0.0275215 & 0.0004433 \\
\hline
\end{tabular}


Table S3 Scaled and real coefficients for the best model obtained for the data set excluding $\mathrm{H}_{2}$ and $\mathrm{HO}$ - (A3B2C1).

\begin{tabular}{ccc}
\hline Parameter & Scaled coefficient & Real coefficient \\
\hline intercept & 0.010702 & -14.3304 \\
x3 & 0.107007 & 0.484081 \\
x8 & 0.151839 & 0.164672 \\
x9 & -0.03308 & -0.01067 \\
x1*x7 & 0.294707 & 0.003223 \\
x1*x9 & 0.127356 & 0.004145 \\
x2*x5 & -0.22764 & -0.09666 \\
x2*x6 & -0.10331 & -0.00235 \\
x2*x9 & -0.20979 & -0.0235 \\
x4*8 & 0.186132 & 0.135114 \\
x5*x6 & 0.136066 & 0.000737 \\
x5*8 & 0.192965 & 0.011867 \\
x6*x9 & 0.064007 & $9.99 \mathrm{E}-05$ \\
x8*x9 & 0.035087 & 0.000516 \\
\hline
\end{tabular}

\section{Individual models for $\mathrm{H}_{2}$ and $\mathrm{HO}$ -}

The heats of adsorption for $\mathrm{H}_{2}$ and $\mathrm{HO}$ - have been identified as potential outliers in the models describing multiple metals an adsorptives. To demonstrate that the descriptors proposed here are still valid to describe the adsorption of these species, they have been modeled separately using the best combination of descriptors identified (Table S3). Since in this case a model only describes a single adsorptive species, descriptors related to the adsorptive $(x 7, x 8$ and $x 9)$ have been discarded. The modeling results for $\mathrm{H}_{2}\left(R^{2}=0.95\right.$ and RMSEE $\left.=0.14 \mathrm{eV}\right)$ and $\mathrm{HO} \cdot\left(R^{2}=0.91\right.$ and RMSEE $\left.=0.48 \mathrm{eV}\right)$ are shown in Figure $\mathrm{S} 7$ and Figure $\mathrm{S} 8$.

Table S4 Model coefficients in real scale for the individual models describing heat of adsorption of $\mathrm{H}_{2}$ and $\mathrm{HO}$ -

\begin{tabular}{ccc}
\hline Parameter & Model for $\mathrm{H}_{\mathbf{2}}$ & Model for HO- \\
\hline intercept & 0.8496234 & 2.9236674 \\
$\mathbf{x 3}$ & -0.1654974 & -0.4563958 \\
$\mathbf{\text { x2*x5 }}$ & -0.0175603 & -0.1530765 \\
$\mathbf{\text { x2*x6 }}$ & -0.0037634 & -0.0022056 \\
$\mathbf{\text { x5*x6 }}$ & 0.0010038 & 0.0020596 \\
\hline
\end{tabular}




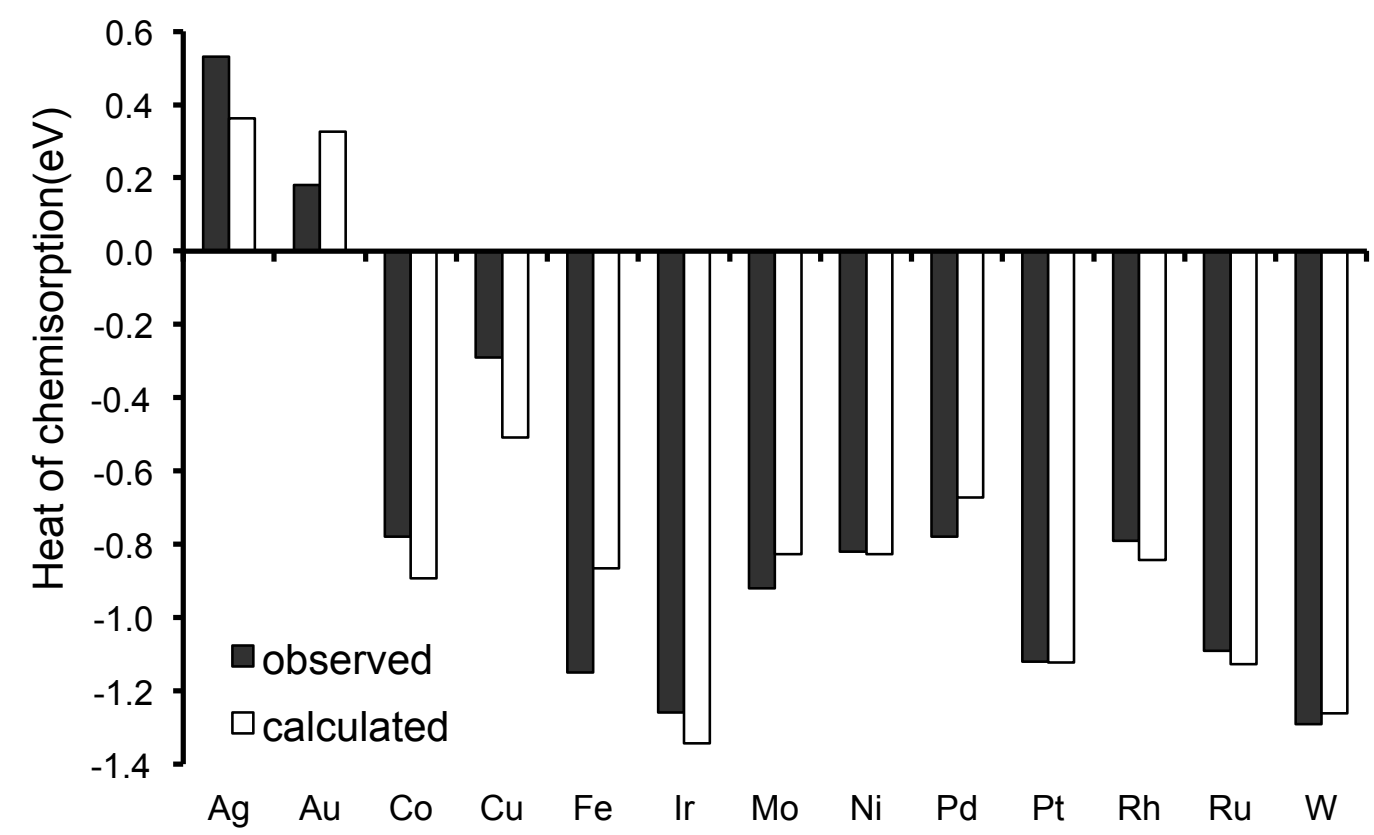

Figure S7 Chemisorption energies of hydrogen derived from DFT (solid bars) and calculated by descriptor model (open bars) for the 13 metals considered.

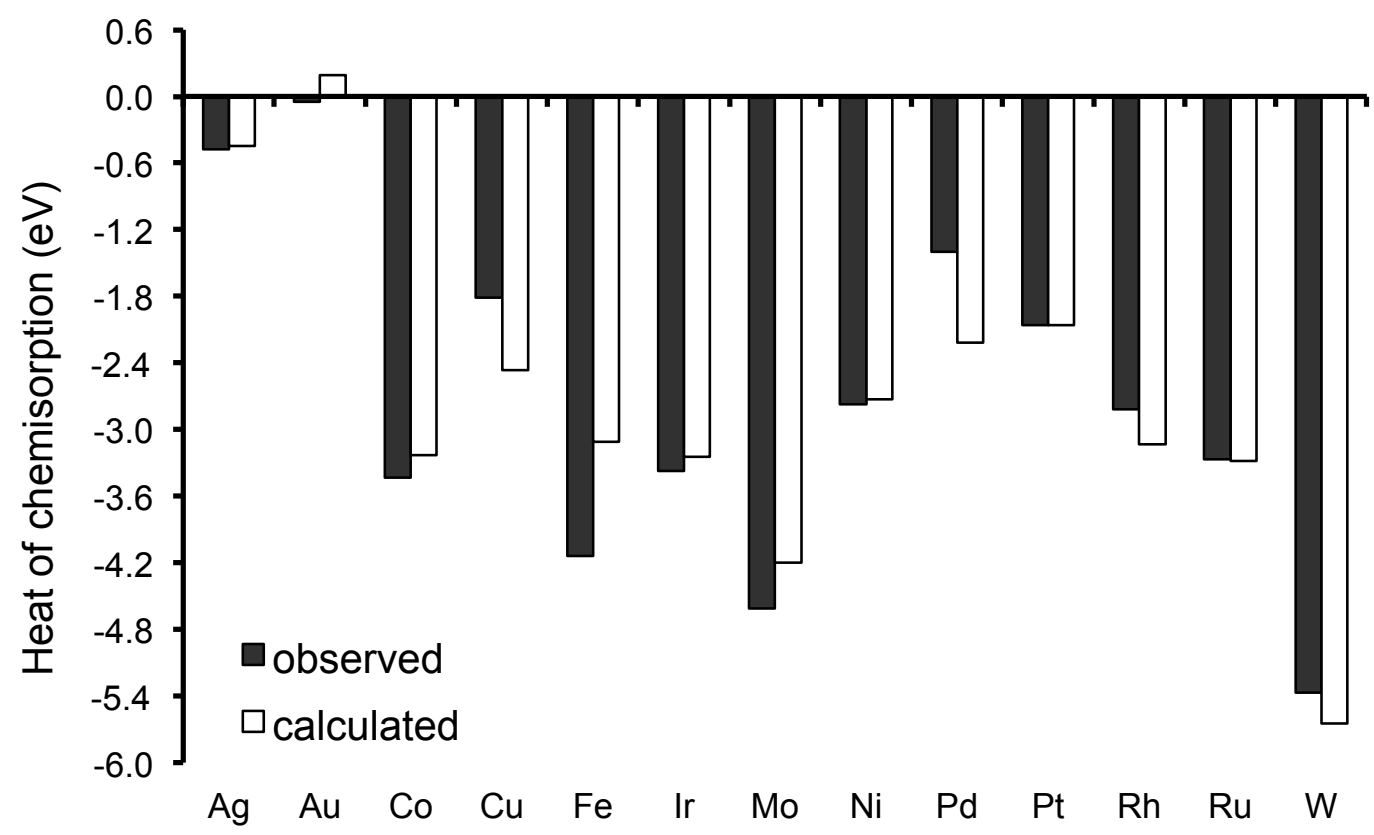

Figure S8 Chemisorption energies of hydroxyl radical derived from DFT (solid bars) and calculated by descriptor model (open bars) for the 13 metals considered. 


\section{Detailed modeling results}

This section contains the modeling results detailed to a level sufficient to reproduce any of the models discussed in the main text ${ }^{1}$. In the diagram below, the work flow for reproducing a scenario of choice is shown. One simply needs to combine information from Table S5 (data set), Table S6 (variable selection) and (training set selection) and regress a PLS model using one's software of choice to reproduce the models from the main text (main text Table 2). Using an appropriate implementation of the PLS algorithm, regression of a model with the same number of latent variables as indicated in the text will produce a model of similar quality and stability. Due to small differences in the various implementations of the PLS algorithm, small numerical differences in the results can be expected. One aspect in particular to keep in mind is scaling. After partitioning the data into a training and a test set, the training set should be scaled to zero mean and unit variance ${ }^{2}$. The column means and standard deviation obtained in scaling the training set should then be used to scale the validation set. Most commercial software packages will do this automatically.

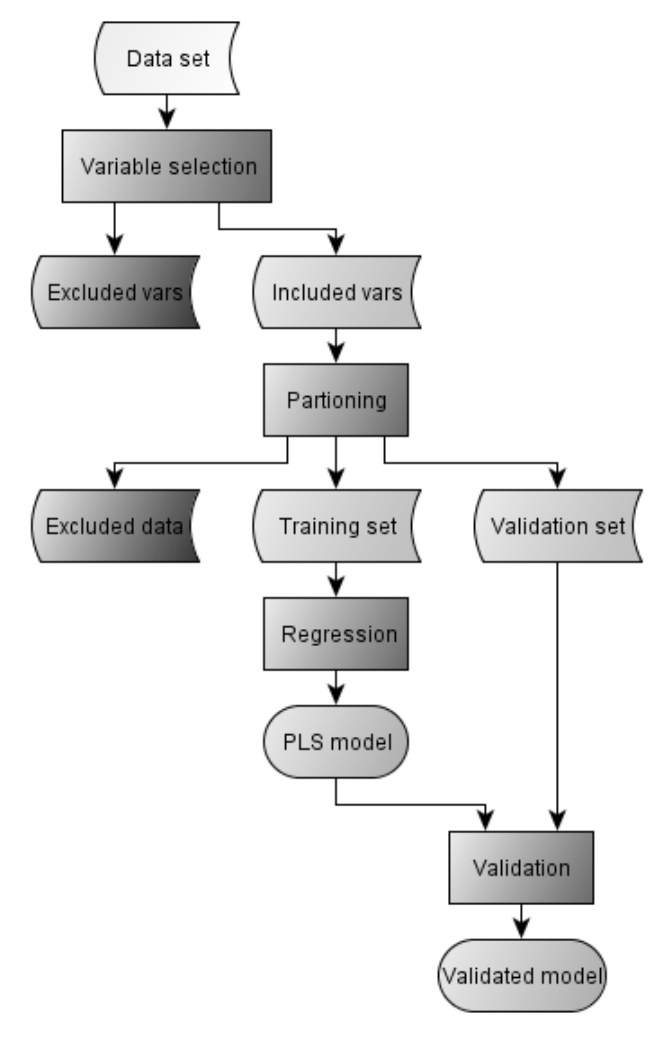

Diagram 1 Flow chart describing the workflow to reproduce any modeling scenario from the main text

\footnotetext{
${ }^{1}$ To facilitate the use of the modeling results the tables from this document can also be obtained in Microsoft Excel format from the publishers' website.

${ }^{2}$ To scale the training set, first calculate the average and standard deviation for each column. The training set data is then scaled by first subtracting the column mean and then dividing by column standard deviation for each entry in a column. To achieve the same scaling for the validation set, apply the procedure above to the validation set using the column means and standard deviations obtained for the training set.
} 
Table S5 Heat of adsorption (y) and descriptor values ( $x 1-x 9)$ for all 130 metal-adsorptive pairs

\begin{tabular}{|c|c|c|c|c|c|c|c|c|c|c|c|c|}
\hline ID & Metal & Adsorptive & $\bar{Y}$ & $\mathrm{x1}$ & $\mathrm{x} 2$ & $x 3$ & $\mathrm{x4}$ & $\times 5$ & $x 6$ & $x 7$ & \begin{tabular}{|l|}
$x 8$ \\
\end{tabular} & $\times 9$ \\
\hline 1 & $\mathrm{Fe}$ & $\mathrm{H}_{2}$ & -1.15 & 6 & 2.45 & 7.902 & 1.25 & 8.18 & 55.85 & 86.98 & 22.82 & 2 \\
\hline 2 & $\mathrm{Fe}$ & $\mathrm{HO} \cdot$ & -4.14 & 6 & 2.45 & 7.902 & 1.25 & 8.18 & 55.85 & 106.24 & 21.05 & 17 \\
\hline 3 & $\mathrm{Fe}$ & $\mathrm{N}_{2}$ & -1.27 & 6 & 2.45 & 7.902 & 1.25 & 8.18 & 55.85 & 139.66 & 21.79 & 28 \\
\hline 4 & $\mathrm{Fe}$ & $\mathrm{CO}$ & -2.53 & 6 & 2.45 & 7.902 & 1.25 & 8.18 & 55.85 & 147.35 & 19.43 & 28 \\
\hline 5 & $\mathrm{Fe}$ & NO & -4.66 & 6 & 2.45 & 7.902 & 1.25 & 8.18 & 55.85 & 134.87 & 14.07 & 30 \\
\hline 6 & $\mathrm{Fe}$ & $\mathrm{O}_{2}$ & -6.3 & 6 & 2.45 & 7.902 & 1.25 & 8.18 & 55.85 & 122.71 & 13.51 & 32 \\
\hline 7 & $\mathrm{Fe}$ & $\mathrm{H}_{2} \mathrm{O}$ & -1.98 & 6 & 2.45 & 7.902 & 1.25 & 8.18 & 55.85 & 118.53 & 19.4 & 18 \\
\hline 8 & $\mathrm{Fe}$ & $\mathrm{CO}_{2}$ & -2.51 & 6 & 2.45 & 7.902 & 1.25 & 8.18 & 55.85 & 165.03 & 20.96 & 44 \\
\hline 9 & $\mathrm{Fe}$ & $\mathrm{NH}_{3}$ & -1.45 & 6 & 2.45 & 7.902 & 1.25 & 8.18 & 55.85 & 138.04 & 17.48 & 17 \\
\hline 10 & $\mathrm{Fe}$ & $\mathrm{CH}_{4}$ & -1.07 & 6 & 2.45 & 7.902 & 1.25 & 8.18 & 55.85 & 155.79 & 21.82 & 16 \\
\hline 11 & Co & $\mathrm{H}_{2}$ & -0.78 & 7 & 2.53 & 7.881 & 1.26 & 8.38 & 58.93 & 86.98 & 22.82 & 2 \\
\hline 12 & Co & $\mathrm{HO} \cdot$ & -3.43 & 7 & 2.53 & 7.881 & 1.26 & 8.38 & 58.93 & 106.24 & 21.05 & 17 \\
\hline 13 & Co & $\mathrm{N}_{2}$ & -0.38 & 7 & 2.53 & 7.881 & 1.26 & 8.38 & 58.93 & 139.66 & 21.79 & 28 \\
\hline 14 & Co & $\mathrm{CO}$ & -1.51 & 7 & 2.53 & 7.881 & 1.26 & 8.38 & 58.93 & 147.35 & 19.43 & 28 \\
\hline 15 & Co & NO & -3.63 & 7 & 2.53 & 7.881 & 1.26 & 8.38 & 58.93 & 134.87 & 14.07 & 30 \\
\hline 16 & Co & $\mathrm{O}_{2}$ & -5.07 & 7 & 2.53 & 7.881 & 1.26 & 8.38 & 58.93 & 122.71 & 13.51 & 32 \\
\hline 17 & Co & $\mathrm{H}_{2} \mathrm{O}$ & -0.99 & 7 & 2.53 & 7.881 & 1.26 & 8.38 & 58.93 & 118.53 & 19.4 & 18 \\
\hline 18 & Co & $\mathrm{CO}_{2}$ & -0.83 & 7 & 2.53 & 7.881 & 1.26 & 8.38 & 58.93 & 165.03 & 20.96 & 44 \\
\hline 19 & Co & $\mathrm{NH}_{3}$ & -0.43 & 7 & 2.53 & 7.881 & 1.26 & 8.38 & 58.93 & 138.04 & 17.48 & 17 \\
\hline 20 & Co & $\mathrm{CH}_{4}$ & 0.09 & 7 & 2.53 & 7.881 & 1.26 & 8.38 & 58.93 & 155.79 & 21.82 & 16 \\
\hline 21 & $\mathrm{Ni}$ & $\mathrm{H}_{2}$ & -0.82 & 8 & 2.42 & 7.64 & 1.21 & 7.42 & 58.69 & 86.98 & 22.82 & 2 \\
\hline 22 & $\mathrm{Ni}$ & $\mathrm{HO} \cdot$ & -2.77 & 8 & 2.42 & 7.64 & 1.21 & 7.42 & 58.69 & 106.24 & 21.05 & 17 \\
\hline 23 & $\mathrm{Ni}$ & $\mathrm{N}_{2}$ & -0.1 & 8 & 2.42 & 7.64 & 1.21 & 7.42 & 58.69 & 139.66 & 21.79 & 28 \\
\hline 24 & $\mathrm{Ni}$ & $\mathrm{CO}$ & -1.05 & 8 & 2.42 & 7.64 & 1.21 & 7.42 & 58.69 & 147.35 & 19.43 & 28 \\
\hline 25 & $\mathrm{Ni}$ & $\mathrm{NO}$ & -2.87 & 8 & 2.42 & 7.64 & 1.21 & 7.42 & 58.69 & 134.87 & $\begin{array}{l}14.07 \\
\end{array}$ & 30 \\
\hline 26 & $\mathrm{Ni}$ & $\mathrm{O}_{2}$ & -3.9 & 8 & 2.42 & 7.64 & 1.21 & 7.42 & 58.69 & 122.71 & 13.51 & 32 \\
\hline 27 & $\mathrm{Ni}$ & $\mathrm{H}_{2} \mathrm{O}$ & -0.45 & 8 & 2.42 & 7.64 & 1.21 & 7.42 & 58.69 & 118.53 & 19.4 & 18 \\
\hline 28 & $\mathrm{Ni}$ & $\mathrm{CO}_{2}$ & 0.17 & 8 & 2.42 & 7.64 & 1.21 & 7.42 & 58.69 & 165.03 & 20.96 & 44 \\
\hline 29 & $\mathrm{Ni}$ & $\mathrm{NH}_{3}$ & -0.37 & 8 & 2.42 & 7.64 & 1.21 & 7.42 & 58.69 & 138.04 & 17.48 & 17 \\
\hline 30 & $\mathrm{Ni}$ & $\mathrm{CH}_{4}$ & -0.13 & 8 & 2.42 & 7.64 & 1.21 & 7.42 & 58.69 & 155.79 & 21.82 & 16 \\
\hline 31 & $\mathrm{Cu}$ & $\mathrm{H}_{2}$ & -0.29 & 10 & 1.81 & 7.726 & 1.38 & 11.01 & 63.55 & 86.98 & 22.82 & 2 \\
\hline 32 & $\mathrm{Cu}$ & $\mathrm{HO} \cdot$ & -1.81 & 10 & 1.81 & 7.726 & 1.38 & 11.01 & 63.55 & 106.24 & 21.05 & 17 \\
\hline 33 & $\mathrm{Cu}$ & $\mathrm{N}_{2}$ & 2.88 & 10 & 1.81 & 7.726 & 1.38 & 11.01 & 63.55 & 139.66 & 21.79 & 28 \\
\hline 34 & $\mathrm{Cu}$ & $\mathrm{CO}$ & 1.77 & 10 & 1.81 & 7.726 & 1.38 & 11.01 & 63.55 & 147.35 & 19.43 & 28 \\
\hline 35 & $\mathrm{Cu}$ & $\mathrm{NO}$ & -0.68 & 10 & 1.81 & 7.726 & 1.38 & 11.01 & 63.55 & 134.87 & 14.07 & 30 \\
\hline 36 & $\mathrm{Cu}$ & $\mathrm{O}_{2}$ & -2.51 & 10 & 1.81 & 7.726 & 1.38 & 11.01 & 63.55 & 122.71 & 13.51 & 32 \\
\hline 37 & $\mathrm{Cu}$ & $\mathrm{H}_{2} \mathrm{O}$ & 0.78 & 10 & 1.81 & 7.726 & 1.38 & 11.01 & 63.55 & 118.53 & 19.4 & 18 \\
\hline 38 & $\mathrm{Cu}$ & $\mathrm{CO}_{2}$ & 3.69 & 10 & 1.81 & 7.726 & 1.38 & 11.01 & 63.55 & 165.03 & 20.96 & 44 \\
\hline 39 & $\mathrm{Cu}$ & $\mathrm{NH}_{3}$ & 1.92 & 10 & 1.81 & 7.726 & 1.38 & 11.01 & 63.55 & 138.04 & 17.48 & 17 \\
\hline 40 & $\mathrm{Cu}$ & $\mathrm{CH}_{4}$ & 3.06 & 10 & 1.81 & 7.726 & 1.38 & 11.01 & 63.55 & 155.79 & 21.82 & 16 \\
\hline 41 & Mo & $\mathrm{H}_{2}$ & -0.92 & 5 & 2.96 & 7.092 & 1.45 & 12.77 & 95.94 & 86.98 & 22.82 & 2 \\
\hline 42 & Mo & $\mathrm{HO} \cdot$ & -4.61 & 5 & 2.96 & 7.092 & 1.45 & 12.77 & 95.94 & 106.24 & 21.05 & 17 \\
\hline 43 & Mo & $\mathrm{N}_{2}$ & -2.76 & 5 & 2.96 & 7.092 & 1.45 & 12.77 & 95.94 & 139.66 & 21.79 & 28 \\
\hline 44 & Mo & $\mathrm{CO}$ & -3.61 & 5 & 2.96 & 7.092 & 1.45 & 12.77 & 95.94 & 147.35 & 19.43 & 28 \\
\hline 45 & Mo & NO & -5.99 & 5 & 2.96 & 7.092 & 1.45 & 12.77 & 95.94 & 134.87 & 14.07 & 30 \\
\hline 46 & Mo & $\mathrm{O}_{2}$ & $\begin{array}{l}-7.48 \\
\end{array}$ & 5 & 2.96 & 7.092 & 1.45 & 12.77 & 95.94 & 122.71 & 13.51 & 32 \\
\hline 47 & Mo & $\mathrm{H}_{2} \mathrm{O}$ & -2.33 & 5 & 2.96 & 7.092 & 1.45 & 12.77 & 95.94 & 118.53 & 19.4 & 18 \\
\hline 48 & Mo & $\mathrm{CO}_{2}$ & -4.18 & 5 & 2.96 & 7.092 & 1.45 & 12.77 & 95.94 & 165.03 & 20.96 & 44 \\
\hline 49 & Mo & $\mathrm{NH}_{3}$ & -1.84 & 5 & 2.96 & 7.092 & 1.45 & 12.77 & 95.94 & 138.04 & 17.48 & 17 \\
\hline 50 & Mo & $\mathrm{CH}_{4}$ & $\begin{array}{l}-1.09 \\
\end{array}$ & 5 & 2.96 & 7.092 & 1.45 & 12.77 & 95.94 & 155.79 & 21.82 & 16 \\
\hline 51 & $\mathrm{Ru}$ & $\mathrm{H}_{2}$ & $\begin{array}{c}1.09 \\
\end{array}$ & 7 & 3.05 & 7.36 & 1.26 & 8.38 & 101.07 & 86.98 & 22.82 & 2 \\
\hline 52 & $\mathrm{Ru}$ & $\mathrm{HO} \cdot$ & -3.27 & 7 & 3.05 & 7.36 & 1.26 & 8.38 & 101.07 & 106.24 & 21.05 & 17 \\
\hline 53 & $\mathrm{Ru}$ & $\mathrm{N}_{2}$ & -0.84 & 7 & 3.05 & 7.36 & 1.26 & 8.38 & 101.07 & 139.66 & 21.79 & 28 \\
\hline 54 & $\mathrm{Ru}$ & $\mathrm{CO}$ & -1.62 & 7 & 3.05 & 7.36 & 1.26 & 8.38 & 101.07 & 147.35 & 19.43 & 28 \\
\hline 55 & $\mathrm{Ru}$ & $\mathrm{NO}$ & -3.6 & 7 & 3.05 & 7.36 & 1.26 & 8.38 & 101.07 & 134.87 & 14.07 & 30 \\
\hline 56 & $\mathrm{Ru}$ & $\mathrm{O}_{2}$ & -4.62 & 7 & 3.05 & 7.36 & 1.26 & 8.38 & 101.07 & 122.71 & 13.51 & 32 \\
\hline 57 & $\mathrm{Ru}$ & $\mathrm{H}_{2} \mathrm{O}$ & $\begin{array}{l}-1.08 \\
\end{array}$ & 7 & 3.05 & 7.36 & 1.26 & 8.38 & 101.07 & 118.53 & 19.4 & 18 \\
\hline 58 & $\mathrm{Ru}$ & $\mathrm{CO}_{2}$ & -0.77 & 7 & 3.05 & 7.36 & 1.26 & 8.38 & 101.07 & 165.03 & 20.96 & 44 \\
\hline 59 & $\mathrm{Ru}$ & $\mathrm{NH}_{3}$ & -1.14 & 7 & 3.05 & 7.36 & 1.26 & 8.38 & 101.07 & 138.04 & 17.48 & 17 \\
\hline 60 & $\mathrm{Ru}$ & $\mathrm{CH}_{4}$ & -0.88 & 7 & 3.05 & 7.36 & 1.26 & 8.38 & 101.07 & 155.79 & 21.82 & 16 \\
\hline 61 & $\mathrm{Rh}$ & $\mathrm{H}_{2}$ & -0.79 & 8 & 2.68 & 7.459 & 1.35 & 10.31 & 102.91 & 86.98 & 22.82 & 2 \\
\hline
\end{tabular}




\begin{tabular}{|c|c|c|c|c|c|c|c|c|c|c|c|c|}
\hline ID & Metal & Adsorptive & $y$ & $x 1$ & $x 2$ & $x 3$ & $x 4$ & $x 5$ & $\mathrm{x6}$ & $x 7$ & $x 8$ & $\times 9$ \\
\hline 63 & $\mathrm{Rh}$ & $\mathrm{N}_{2}$ & -0.7 & 8 & 2.68 & 7.459 & 1.35 & 10.31 & 102.91 & 139.66 & 21.79 & 28 \\
\hline 64 & $\mathrm{Rh}$ & $\mathrm{CO}$ & -1.12 & 8 & 2.68 & 7.459 & 1.35 & 10.31 & 102.91 & 147.35 & 19.43 & 28 \\
\hline 65 & $\mathrm{Rh}$ & $\mathrm{NO}$ & -3.23 & 8 & 2.68 & 7.459 & 1.35 & 10.31 & 102.91 & 134.87 & 14.07 & 30 \\
\hline 66 & $\mathrm{Rh}$ & $\mathrm{O}_{2}$ & -4.03 & 8 & 2.68 & 7.459 & 1.35 & 10.31 & 102.91 & 122.71 & 13.51 & 32 \\
\hline 67 & $\mathrm{Rh}$ & $\mathrm{H}_{2} \mathrm{O}$ & -0.48 & 8 & 2.68 & 7.459 & 1.35 & 10.31 & 102.91 & 118.53 & 19.4 & 18 \\
\hline 68 & $\mathrm{Rh}$ & $\mathrm{CO}_{2}$ & 0.03 & 8 & 2.68 & 7.459 & 1.35 & 10.31 & 102.91 & 165.03 & 20.96 & 44 \\
\hline 69 & $\mathrm{Rh}$ & $\mathrm{NH}_{3}$ & -0.61 & 8 & 2.68 & 7.459 & 1.35 & 10.31 & 102.91 & 138.04 & 17.48 & 17 \\
\hline 70 & $\mathrm{Rh}$ & $\mathrm{CH}_{4}$ & -0.06 & 8 & 2.68 & 7.459 & 1.35 & 10.31 & 102.91 & 155.79 & 21.82 & 16 \\
\hline 71 & $\mathrm{Pd}$ & $\mathrm{H}_{2}$ & -0.78 & 10 & 2.03 & 8.337 & 1.31 & 9.42 & 106.42 & 86.98 & 22.82 & 2 \\
\hline 72 & $P d$ & $\mathrm{HO}$. & -1.4 & 10 & 2.03 & 8.337 & 1.31 & 9.42 & 106.42 & 106.24 & 21.05 & 17 \\
\hline 73 & $\mathrm{Pd}$ & $\mathrm{N}_{2}$ & 1.78 & 10 & 2.03 & 8.337 & 1.31 & 9.42 & 106.42 & 139.66 & 21.79 & 28 \\
\hline 74 & $\mathrm{Pd}$ & $\mathrm{CO}$ & 0.38 & 10 & 2.03 & 8.337 & 1.31 & 9.42 & 106.42 & 147.35 & 19.43 & 28 \\
\hline 75 & $P d$ & NO & -0.58 & 10 & 2.03 & 8.337 & 1.31 & 9.42 & 106.42 & 134.87 & 14.07 & 30 \\
\hline 76 & $\mathrm{Pd}$ & $\mathrm{O}_{2}$ & -1.2 & 10 & 2.03 & 8.337 & 1.31 & 9.42 & 106.42 & 122.71 & 13.51 & 32 \\
\hline 77 & $\mathrm{Pd}$ & $\mathrm{H}_{2} \mathrm{O}$ & 0.95 & 10 & 2.03 & 8.337 & 1.31 & 9.42 & 106.42 & 118.53 & 19.4 & 18 \\
\hline 78 & $\mathrm{Pd}$ & $\mathrm{CO}_{2}$ & 2.96 & 10 & 2.03 & 8.337 & 1.31 & 9.42 & 106.42 & 165.03 & 20.96 & 44 \\
\hline 79 & $\mathrm{Pd}$ & $\mathrm{NH}_{3}$ & 0.64 & 10 & 2.03 & 8.337 & 1.31 & 9.42 & 106.42 & 138.04 & 17.48 & 17 \\
\hline 80 & $\mathrm{Pd}$ & $\mathrm{CH}_{4}$ & 0.04 & 10 & 2.03 & 8.337 & 1.31 & 9.42 & 106.42 & 155.79 & 21.82 & 16 \\
\hline 81 & $\mathrm{Ag}$ & $\mathrm{H}_{2}$ & 0.53 & 10 & 1.28 & 7.576 & 1.53 & 15 & 107.87 & 86.98 & 22.82 & 2 \\
\hline 82 & $\mathrm{Ag}$ & $\mathrm{HO}$. & -0.48 & 10 & 1.28 & 7.576 & 1.53 & 15 & 107.87 & 106.24 & 21.05 & 17 \\
\hline 83 & $\mathrm{Ag}$ & $\mathrm{N}_{2}$ & 5.86 & 10 & 1.28 & 7.576 & 1.53 & 15 & 107.87 & 139.66 & 21.79 & 28 \\
\hline 84 & $\mathrm{Ag}$ & $\mathrm{CO}$ & 4.32 & 10 & 1.28 & 7.576 & 1.53 & 15 & 107.87 & 147.35 & 19.43 & 28 \\
\hline 85 & $\mathrm{Ag}$ & $\mathrm{NO}$ & 1.73 & 10 & 1.28 & 7.576 & 1.53 & 15 & 107.87 & 134.87 & 14.07 & 30 \\
\hline 86 & $\mathrm{Ag}$ & $\mathrm{O}_{2}$ & -0.65 & 10 & 1.28 & 7.576 & 1.53 & 15 & 107.87 & 122.71 & 13.51 & 32 \\
\hline 87 & $\mathrm{Ag}$ & $\mathrm{H}_{2} \mathrm{O}$ & 2.52 & 10 & 1.28 & 7.576 & 1.53 & 15 & 107.87 & 118.53 & 19.4 & 18 \\
\hline 88 & $\mathrm{Ag}$ & $\mathrm{CO}_{2}$ & 7.16 & 10 & 1.28 & 7.576 & 1.53 & 15 & 107.87 & 165.03 & 20.96 & 44 \\
\hline 89 & $\mathrm{Ag}$ & $\mathrm{NH}_{3}$ & 4.63 & 10 & 1.28 & 7.576 & 1.53 & 15 & 107.87 & 138.04 & 17.48 & 17 \\
\hline 90 & $\mathrm{Ag}$ & $\mathrm{CH}_{4}$ & 6.31 & 10 & 1.28 & 7.576 & 1.53 & 15 & 107.87 & 155.79 & 21.82 & 16 \\
\hline 91 & $\mathrm{~W}$ & $\mathrm{H}_{2}$ & -1.29 & 4 & 3.47 & 7.98 & 1.46 & 13.04 & 103.84 & 86.98 & 22.82 & 2 \\
\hline 92 & $\mathrm{~W}$ & $\mathrm{HO} \cdot$ & -5.37 & 4 & 3.47 & 7.98 & 1.46 & 13.04 & 103.84 & 106.24 & 21.05 & 17 \\
\hline 93 & $\mathrm{~W}$ & $\mathrm{~N}_{2}$ & -4.33 & 4 & 3.47 & 7.98 & 1.46 & 13.04 & 103.84 & 139.66 & 21.79 & 28 \\
\hline 94 & W & $\mathrm{CO}$ & -4.73 & 4 & 3.47 & 7.98 & 1.46 & 13.04 & 103.84 & 147.35 & 19.43 & 28 \\
\hline 95 & W & $\mathrm{NO}$ & -7.34 & 4 & 3.47 & 7.98 & 1.46 & 13.04 & 103.84 & 134.87 & 14.07 & 30 \\
\hline 96 & W & $\mathrm{O}_{2}$ & -8.62 & 4 & 3.47 & 7.98 & 1.46 & 13.04 & 103.84 & 122.71 & 13.51 & 32 \\
\hline 97 & $\mathrm{~W}$ & $\mathrm{H}_{2} \mathrm{O}$ & -3.27 & 4 & 3.47 & 7.98 & 1.46 & 13.04 & 103.84 & 118.53 & 19.4 & 18 \\
\hline 98 & $\mathrm{~W}$ & $\mathrm{CO}_{2}$ & -5.87 & 4 & 3.47 & 7.98 & 1.46 & 13.04 & 103.84 & 165.03 & 20.96 & 44 \\
\hline 99 & W & $\mathrm{NH}_{3}$ & -3.18 & 4 & 3.47 & 7.98 & 1.46 & 13.04 & 103.84 & 138.04 & 17.48 & 17 \\
\hline 100 & $\mathrm{~W}$ & $\mathrm{CH}_{4}$ & -2.37 & 4 & 3.47 & 7.98 & 1.46 & 13.04 & 103.84 & 155.79 & 21.82 & 16 \\
\hline 101 & $\mathrm{Ir}$ & $\mathrm{H}_{2}$ & -1.26 & 7 & 3.03 & 9.1 & 1.37 & 10.77 & 192.22 & 86.98 & 22.82 & 2 \\
\hline 102 & Ir & $\mathrm{HO}$ & -3.37 & 7 & 3.03 & 9.1 & 1.37 & 10.77 & 192.22 & 106.24 & 21.05 & 17 \\
\hline 103 & Ir & $\mathrm{N}_{2}$ & -0.59 & 7 & 3.03 & 9.1 & 1.37 & 10.77 & 192.22 & 139.66 & 21.79 & 28 \\
\hline 104 & Ir & $\mathrm{CO}$ & -1.07 & 7 & 3.03 & 9.1 & 1.37 & 10.77 & 192.22 & 147.35 & 19.43 & 28 \\
\hline 105 & Ir & $\mathrm{NO}$ & -3.49 & 7 & 3.03 & 9.1 & 1.37 & 10.77 & 192.22 & 134.87 & 14.07 & 30 \\
\hline 106 & Ir & $\mathrm{O}_{2}$ & -4.65 & 7 & 3.03 & 9.1 & 1.37 & 10.77 & 192.22 & 122.71 & 13.51 & 32 \\
\hline 107 & Ir & $\mathrm{H}_{2} \mathrm{O}$ & -1.26 & 7 & 3.03 & 9.1 & 1.37 & 10.77 & 192.22 & 118.53 & 19.4 & 18 \\
\hline 108 & Ir & $\mathrm{CO}_{2}$ & -0.23 & 7 & 3.03 & 9.1 & 1.37 & 10.77 & 192.22 & 165.03 & 20.96 & 44 \\
\hline 109 & Ir & $\mathrm{NH}_{3}$ & -1.27 & 7 & 3.03 & 9.1 & 1.37 & 10.77 & 192.22 & 138.04 & 17.48 & 17 \\
\hline 110 & Ir & $\mathrm{CH}_{4}$ & -0.65 & 7 & 3.03 & 9.1 & 1.37 & 10.77 & 192.22 & 155.79 & 21.82 & 16 \\
\hline 111 & $\mathrm{Pt}$ & $\mathrm{H}_{2}$ & -1.12 & 9 & 2.48 & 9 & 1.28 & 8.78 & 195.08 & 86.98 & 22.82 & 2 \\
\hline 112 & $\mathrm{Pt}$ & $\mathrm{HO}$ & -2.06 & 9 & 2.48 & 9 & 1.28 & 8.78 & 195.08 & 106.24 & 21.05 & 17 \\
\hline 113 & $\mathrm{Pt}$ & $\mathrm{N}_{2}$ & 1.37 & 9 & 2.48 & 9 & 1.28 & 8.78 & 195.08 & 139.66 & 21.79 & 28 \\
\hline 114 & $\mathrm{Pt}$ & $\mathrm{CO}$ & 0.37 & 9 & 2.48 & 9 & 1.28 & 8.78 & 195.08 & 147.35 & 19.43 & 28 \\
\hline 115 & $\mathrm{Pt}$ & $\mathrm{NO}$ & -1.27 & 9 & 2.48 & 9 & 1.28 & 8.78 & 195.08 & 134.87 & 14.07 & 30 \\
\hline 116 & $\mathrm{Pt}$ & $\mathrm{O}_{2}$ & -2.17 & 9 & 2.48 & 9 & 1.28 & 8.78 & 195.08 & 122.71 & 13.51 & 32 \\
\hline 117 & $\mathrm{Pt}$ & $\mathrm{H}_{2} \mathrm{O}$ & 0.12 & 9 & 2.48 & 9 & 1.28 & 8.78 & 195.08 & 118.53 & 19.4 & 18 \\
\hline 118 & $\mathrm{Pt}$ & $\mathrm{CO}_{2}$ & 2.45 & 9 & 2.48 & 9 & 1.28 & 8.78 & 195.08 & 165.03 & 20.96 & 44 \\
\hline 119 & $\mathrm{Pt}$ & $\mathrm{NH}_{3}$ & -0.08 & 9 & 2.48 & 9 & 1.28 & 8.78 & 195.08 & 138.04 & 17.48 & 17 \\
\hline 120 & $\mathrm{Pt}$ & $\mathrm{CH}_{4}$ & -0.18 & 9 & 2.48 & 9 & 1.28 & 8.78 & 195.08 & 155.79 & 21.82 & 16 \\
\hline 121 & $\mathrm{Au}$ & $\mathrm{H}_{2}$ & 0.18 & 10 & 1.53 & 9.226 & 1.44 & 12.51 & 196.97 & 86.98 & 22.82 & 2 \\
\hline 122 & $\mathrm{Au}$ & $\mathrm{HO}$ & -0.05 & 10 & 1.53 & 9.226 & 1.44 & 12.51 & 196.97 & 106.24 & 21.05 & 17 \\
\hline 123 & $\mathrm{Au}$ & $\mathrm{N}_{2}$ & 5.89 & 10 & 1.53 & 9.226 & 1.44 & 12.51 & 196.97 & 139.66 & 21.79 & 28 \\
\hline 124 & $\mathrm{Au}$ & $\mathrm{CO}$ & 4.58 & 10 & 1.53 & 9.226 & 1.44 & 12.51 & 196.97 & 147.35 & 19.43 & 28 \\
\hline 125 & $\mathrm{Au}$ & $\mathrm{NO}$ & 2.34 & 10 & 1.53 & 9.226 & 1.44 & 12.51 & 196.97 & 134.87 & 14.07 & 30 \\
\hline 126 & $\mathrm{Au}$ & $\mathrm{O}_{2}$ & 0.54 & 10 & 1.53 & 9.226 & 1.44 & 12.51 & 196.97 & 122.71 & 13.51 & 32 \\
\hline 127 & $\mathrm{Au}$ & $\mathrm{H}_{2} \mathrm{O}$ & 2.77 & 10 & 1.53 & 9.226 & 1.44 & 12.51 & 196.97 & 118.53 & 19.4 & 18 \\
\hline
\end{tabular}


Electronic Supplementary Material (ESI) for Physical Chemistry Chemical Physics

This journal is (C) The Owner Societies 2013

Supporting information

\begin{tabular}{|c|c|c|c|c|c|c|c|c|c|c|c|c|}
\hline ID & Metal & Adsorptive & $y$ & $x 1$ & $\times 2$ & $x 3$ & $x 4$ & $\times 5$ & $\times 6$ & $x 7$ & $x 8$ & $x 9$ \\
\hline 128 & $\mathrm{Au}$ & $\mathrm{CO}_{2}$ & 8.02 & 10 & 1.53 & 9.226 & 1.44 & 12.51 & 196.97 & 165.03 & 20.96 & 44 \\
\hline 129 & $\mathrm{Au}$ & $\mathrm{NH}_{3}$ & 4.12 & 10 & 1.53 & 9.226 & 1.44 & 12.51 & 196.97 & 138.04 & 17.48 & 17 \\
\hline 130 & $\mathrm{Au}$ & $\mathrm{CH}_{4}$ & 5.28 & 10 & 1.53 & 9.226 & 1.44 & 12.51 & 196.97 & 155.79 & 21.82 & 16 \\
\hline
\end{tabular}

